\title{
Determinantes sociais do absenteísmo de diabéticos às consultas com
}

\section{endocrinologista}

\author{
Social determinants of absenteeism of diabetic to endocrinologist appointments \\ Determinantes sociales del absentismo de los diabéticos las consultas con un endocrinólogo
}

Recebido: 21/09/2021 | Revisado: 01/10/2021 | Aceito: 04/10/2021 | Publicado: 06/10/2021

Marcelle Lemos Leal

ORCID: https://orcid.org/0000-0001-7878-6654 Universidade Federal do Espírito Santo, Brasil

E-mail marcelle.leal@outlook.com

Edson Theodoro dos Santos Neto

ORCID: https://orcid.org/0000-0002-7351-7719

Universidade Federal do Espírito Santo, Brasil

E-mail: edsontheodoro@uol.com.br

Eliana Zandonade

ORCID: https://orcid.org/ 0000-0001-5160-3280

Universidade Federal do Espírito Santo, Brasil

E-mail: elianazandonade@uol.com.br

Thiago Dias Sarti

ORCID: https://orcid.org/0000-0002-1545-6276

Universidade Federal do Espírito Santo, Brasil E-mail: thiagosarti@yahoo.com.br

Nágela Valadão Cade

ORCID: https://orcid.org/0000-0001-6073-504X

Universidade Federal do Espírito Santo, Brasil E-mail: nagelavc@gmail.com.

\begin{abstract}
Resumo
O objetivo deste estudo é analisar os determinantes sociais de saúde (DSS) associados ao absenteísmo de diabéticos às consultas com o endocrinologista, no Sistema Único de Saúde, no Espírito Santo (ES). Trata-se de estudo transversal por meio de dados primários e secundários com 472 diabéticos $\geq 18$ anos agendados em consulta com endocrinologista no Sistema de Centrais de Regulação do ES. As associações foram avaliadas através de regressão logística hierarquizada agrupadas em cinco níveis: socioeconômico e demográfico, acesso aos serviços de saúde, psicossociais, comportamentais e clínicos do diabetes mellitus (DM). Estiveram associados ao absenteísmo de diabéticos renda domiciliar per capita maior que $\mathrm{R} \$ 499,00$ ( $\mathrm{OR}=1,668$, IC95\%: 1,009-2,756), tempo de espera em dias (OR=0,997, IC95\%: 0,995-0,999), consumo de bebida alcoólica (OR=1,821, IC95\%:1,031-3,239), obesidade (OR=1,773, IC95\%: 1,134-2,773), uso de hipoglicemiante oral e insulina (OR=2,2, IC95\%:1,09-4,44) e ter complicações do DM (OR=2,03, IC95\%:1,297-3,179). O conhecimento dos determinantes sociais do absenteísmo apresentou evidências para avaliação de prioridades e implementação da rede de atenção às pessoas com DM no ES.
\end{abstract}

Palavras-chave: Determinantes sociais da saúde; Absenteísmo; Atenção secundária; Diabetes Mellitus; Agendamento de consultas.

\begin{abstract}
This study aims to analyze the social determinants of health (DSS) associated with absenteeism of diabetics to appointments with an endocrinologist, in the Unified Health System, in Espírito Santo (ES). This is a cross-sectional study using primary and secondary data with 472 diabetics $\geq 18$ years old scheduled in consultation with an endocrinologist in the System of Central Regulation of ES. Associations were evaluated using hierarchical logistic regression grouped into five levels: socioeconomic and demographic, access to health services, psychosocial, behavioral and clinical diabetes mellitus (DM). Absenteeism of diabetics was associated with per capita household income greater than $\mathrm{R} \$ 499.00(\mathrm{OR}=1.668,95 \% \mathrm{CI}$ : 1.009-2.756), waiting time in days $(\mathrm{OR}=0.997$, 95\%CI: 0.995$0.999)$, consumption of alcoholic beverages (OR=1.821, 95\%CI: $1.031-3.239)$, obesity $(\mathrm{OR}=1.773$, 95\%CI: $1.134-$ 2.773), use of oral hypoglycemic agents and insulin ( $\mathrm{OR}=2.2,95 \% \mathrm{CI}$ : 1.09-4 .44) and having DM complications $(\mathrm{OR}=2.03$, 95\%CI: 1.297-3.179). Knowledge of the social determinants of absenteeism provided evidence for assessing priorities and implementing the care network for people with DM in ES.
\end{abstract}

Keywords: Social determinants of health; Absenteeism; Secondary care; Diabetes Mellitus; Appointments and schedules. 


\section{Resumen}

El objetivo de este estudio es analizar los determinantes sociales de la salud (DSS) asociados al absentismo de los diabéticos a las consultas con un endocrinólogo, en el Sistema Único de Salud, en Espírito Santo (ES). Se trata de un estudio transversal que utiliza datos primarios y secundarios con 472 diabéticos $\geq 18$ años programados en consulta con un endocrinólogo en el Sistema de Regulación Central de ES. Las asociaciones se evaluaron mediante regresión logística jerárquica agrupada en cinco niveles: socioeconómico y demográfico, acceso a servicios de salud, diabetes mellitus (DM) psicosocial, conductual y clínica. El absentismo de los diabéticos se asoció con un ingreso familiar per cápita superior a $\mathrm{R} \$ 499,00(\mathrm{OR}=1,668$, IC 95\%: 1,009-2,756), tiempo de espera en días $(\mathrm{OR}=0,997$, IC 95\%: 0,995-0,999), consumo de alcohol bebidas (OR = 1,821, 95\% CI: 1,031-3.239), obesidad (OR = 1,773, 95\% CI: 1,134-2,773), uso de hipoglucemiantes orales e insulina (OR =2,2, 95\% CI: 1.09-4. 44) y tener complicaciones de DM $(\mathrm{OR}=2,03$, IC 95\%: 1,297-3,179). El conocimiento de los determinantes sociales del absentismo brindó evidencia para evaluar las prioridades e implementar la red de atención a las personas con DM en ES.

Palabras clave: Determinantes sociales de la salud; Absentismo; Atención secundaria de Salud; Diabetes Mellitus; Citas y horarios.

\section{Introdução}

A elevada prevalência global do diabetes mellitus (DM) (9,3\%) na população de 20 a 79 anos se configura uma importante carga, tanto nos custos diretos para o sistema de saúde e para a sociedade, como nos custos indiretos atribuíveis à mortalidade prematura e às incapacitações temporárias e permanentes decorrentes de suas complicações (International Diabetes Federation [IDF], 2019). Semelhantemente, no Brasil foi estimado que 7,7\% da população acima de 18 anos referiram diagnóstico de DM. Comprometimento da visão (29\%), comprometimento renal (9,5\%), acidente vascular cerebral $(7,2 \%)$ e úlcera nos pés ou amputação de membros $(5,6)$ foram as complicações mais frequentes relatadas pelos diabéticos (Pesquisa Nacional de Saúde [PNS], 2020a).

O controle do DM e suas complicações estão diretamente relacionados ao autogerenciamento, ao acompanhamento regular nos serviços de saúde e às ações das políticas de promoção da saúde (Danaei, et al., 2014). Isso impõe constantes desafios aos gestores da saúde e demais setores governamentais, no tocante ao aumento da demanda por serviços, o custo crescente da assistência médica, a permanente incorporação tecnológica, a ampliação da oferta na Atenção Ambulatorial Especializada (AAE) e a integralidade do cuidado na Atenção Primária à Saúde (APS) (Costa et al., 2017).

Por outro lado, há o absenteísmo, definido como o não comparecimento às consultas (Bender, Molina, \& Melo, 2010; Bittar, Magalhães, Martines, Felizola \& Falcão, 2016), que representa um problema crucial à prestação de cuidados de saúde, reduz a efetividade dos serviços, além de aumentar os custos e os danos aos diabéticos. Ademais, o absenteísmo afeta a assistência e cuidado a outros indivíduos que necessitam de atenção à saúde devido à redução do aproveitamento da oferta da consulta e aumento da fila de espera, uma vez que o indivíduo retorna aos serviços de saúde para um novo agendamento. Também contribui para o desperdício de recursos, ineficiência da clínica e da gestão dos serviços públicos (Bender, Molina, \& Melo, 2010; Mesa, Asencio \& Ruiz, 2015; Bittar, Magalhães, Martines, Felizola \& Falcão, 2016).

O absenteísmo é um problema multicausal que se apresenta nos diferentes contextos e sistemas de saúde e a sua prevalência tem sido relatada em revisões sistemáticas cuja taxa média geral no mundo foi estimada em torno de $23 \%$ nas diferentes especialidades e população (Turkan, et al., 2013; Dantas, Fleck, Oliveira, Hamacher, 2018). No Brasil, estudos revelaram taxas iguais ou superiores a 25\% (Bender, Molina, \& Melo, 2010; Cavalcanti, Cavalcanti, Serrano, Santana, 2013; Bittar, Magalhães, Martines, Felizola \& Falcão, 2016; Beltrame, Oliveira, Santos \& Santos Neto, 2019). Meta-análise com estudos internacionais acerca do absenteísmo de diabéticos às consultas especializadas descreveu que a taxa dos estudos variou de $10 \%$ a $30 \%$ (Brewster, Bartholomew, Holt \& Prince, 2020).

As publicações sobre o absenteísmo às consultas especializadas de diversas patologias apontaram fatores relacionados às diferenças na oferta, organização dos serviços de saúde, nível socioeconômico, barreiras geográficas e de acesso, além de condições comportamentais, emocionais, psicológicas e cognitivas (Bittar, Magalhães, Martines, Felizola \& Falcão, 2016; 
Dantas, Fleck, Oliveira, Hamacher, 2018; Beltrame, Oliveira, Santos, Santos Neto, 2019; Rodrigues, et al., 2019; Farias, Giovanella, Oliveira, Santos Neto, 2019; Farias, Moraes Esposti, Santos Neto, 2020; Brewster, Bartholomew, Holt \& Prince, 2020).

Os fatores identificados nesses estudos, segundo a Organização Mundial de Saúde (OMS), podem estar relacionados “às condições em que as pessoas nascem, vivem, crescem, trabalham e envelhecem” (Organização Mundial de Saúde [OMS], 2011). Dessa forma, é importante considerar que os determinantes sociais de saúde (DSS) estejam atuando como pano de fundo no recrudescimento do absenteísmo às consultas especializadas contribuindo, dessa forma, para aumento das desigualdades em saúde.

Segundo o marco conceitual estabelecido pela OMS em 2011, os DSS são definidos como os mecanismos que geram e reforçam as hierarquias sociais (determinantes estruturais) e, desta forma, impactam circunstâncias materiais, fatores comportamentais, biológicos e psicossociais (determinantes intermediários). Ainda incluem o sistema de saúde, coesão social e capital social (Solar \& Irvin, 2010).

A influência dos DSS na qualidade de vida do diabético pode determinar, em grande parte, os processos de saúde e adoecimento, no que diz respeito, ao estilo de vida, aos hábitos alimentares, a prática de atividade física, adesão à terapêutica, o controle metabólico, o acompanhamento trimestral regular na APS e a consulta especializada, no mínimo, uma vez por ano a partir do diagnóstico (Brasil, 2013).

Diante dessas considerações, o presente estudo tem como objetivo analisar os DSS associados ao absenteísmo de diabéticos às consultas com o endocrinologista, no Sistema Único de Saúde, no estado do Espírito Santo, Brasil.

\section{Materiais e Métodos}

\subsection{Desenho de estudo, fonte e coleta de dados}

Trata-se de estudo transversal (Leopardi; Beck; Nietsche \& Gonzales, 2001) com população de diabéticos maior ou igual a 18 anos, residentes no Espírito Santo (ES) e agendados para consultas com endocrinologista no Sistema Único de Saúde (SUS) no ano de 2018.

Os dados referentes ao agendamento das consultas com endocrinologista realizados em 2018 e as informações de identificação, residência, consultas solicitadas e realizadas dos indivíduos foram extraídas do Sistema de Centrais de Regulação (SISREG), disponibilizados pela Central de Regulação Ambulatorial da Secretaria Estadual do ES. A partir destas informações, foram identificados os indivíduos com diagnóstico referido de diabetes mellitus (DM) conforme a Classificação Internacional de Doenças (CID-10): E10.0 a E12.0, E13.0, E13.1, E14.0, E14.3, E14.4, E14.8, E14.9, G590, G632, G990, H360, O24 e R730. Para o estudo foram elegíveis 4.136 diabéticos com uma prevalência de absenteísmo 37,5\% de acordo com o banco de dados.

No período entre maio de 2019 e janeiro de 2020 foi realizada a coleta de dados primários mediante questionário estruturado aplicado por quatro entrevistadoras habilitadas. Os questionários foram aplicados por meio de ligações telefônicas em dias e horários variados, incluído os finais de semana, e gravadas por meio do aplicativo gratuito de telefonia móvel denominado Automatic Call Recorder.

Foram excluídos os indivíduos que durante a entrevista negaram a condição de diabéticos, estavam institucionalizados; sem condições para responder; recusaram participação; número do telefone ou celular pertencia a outra pessoa ou não atenderam a quatro tentativas de chamadas telefônicas (Figura 1). 


\subsection{População de estudo}

O tamanho amostral foi calculado segundo a prevalência de absenteísmo de $37,5 \%$ e relação de um diabético absenteísta (falta a uma ou mais consultas) para dois diabéticos não absenteístas, nível de significância de 5\%, com poder de teste de $80 \%$, odds ratio (OR) de 1,8 e exposição de $25 \%$ no grupo não absenteísta. Este cálculo foi realizado no aplicativo Statcalc do software Epi Info 7.2.4.0 e resultou em 467 diabéticos.

\subsection{Variáveis de estudo}

Elaborou-se modelo teórico hierarquizado (Figura 2) à luz do marco conceitual dos DSS da OMS (2011).

A variável dependente, absenteísmo, foi confirmada por meio da entrevista e reclassificada quando o diabético: a) foi à consulta, porém, o endocrinologista não compareceu; b) relatou que desconhecia o agendamento da referida consulta; e c) chegou atrasado e não efetuou a consulta. Com isso, 24 diabéticos foram reclassificados após a entrevista e a amostra do estudo correspondeu a 140 diabéticos absenteístas e 332 diabéticos não absenteístas (Figura 1).

No modelo hierárquico as variáveis independentes foram agrupadas em cinco níveis. Nível 1 - fatores socioeconômicos e demográficos: sexo, faixa etária, raça/cor, escolaridade, situação conjugal, zona de moradia, renda média domiciliar per capita (R\$ 998,00 - o salário mínimo vigente no período do estudo), quem é o chefe da família, escolaridade do chefe da família, benefício social (bolsa família ou benefício prestação continuada); água tratada, esgoto ligado na rede e recolhimento de lixo pelo serviço público. Nível 2 - acesso aos serviços de saúde: tempo de espera em dias entre a solicitação e o agendamento da consulta com o endocrinologista e plano de saúde. Nível 3 - fatores psicossociais: escala de suporte social (apoio material e apoio social) com $\alpha$ de Cronbach $\geq 0,83$ (Sherbourne, Stewart, 1991; Griep, Chor, Faerstein, Werneck, Lopes, 2005), escala de vizinhança (coesão social, segurança e vitimização pessoal) com coeficiente de correlação intraclasse, respectivamente, 0,86, 0,83 e 0,66 (Mujahid, Roux, Morenoff \& Raghunathan, 2007), discriminação percebida e qualidade de saúde percebida. Para classificar as dimensões que compõe o suporte social e a vizinhança calculou-se a razão (total de pontos obtidos/pontuação máxima da dimensão) multiplicado por 100. Em seguida, categorizou-se as pontuações em tercis de tamanhos aproximadamente iguais (Griep, Chor, Faerstein, Lopes, 2003; Oliveira, et al., 2011; Secretti, et al., 2019). Nível 4 fatores comportamentais: hábito de fumar, hábito de consumir bebida alcoólica e índice de massa corporal (IMC) de acordo com os escores propostos pela World Health Organization Expert Committee (1995). Nível 5 - fatores clínicos do DM: tipo de medicamento em uso, complicações, comorbidades, limitações ou deficiência. 
Figura 1. Descrição das etapas para composição da amostra e coleta de dados por meio de entrevista telefônica dos diabéticos cadastrados no Sistema de Centrais de Regulação (SISREG). Espírito Santo, ES, 2018.

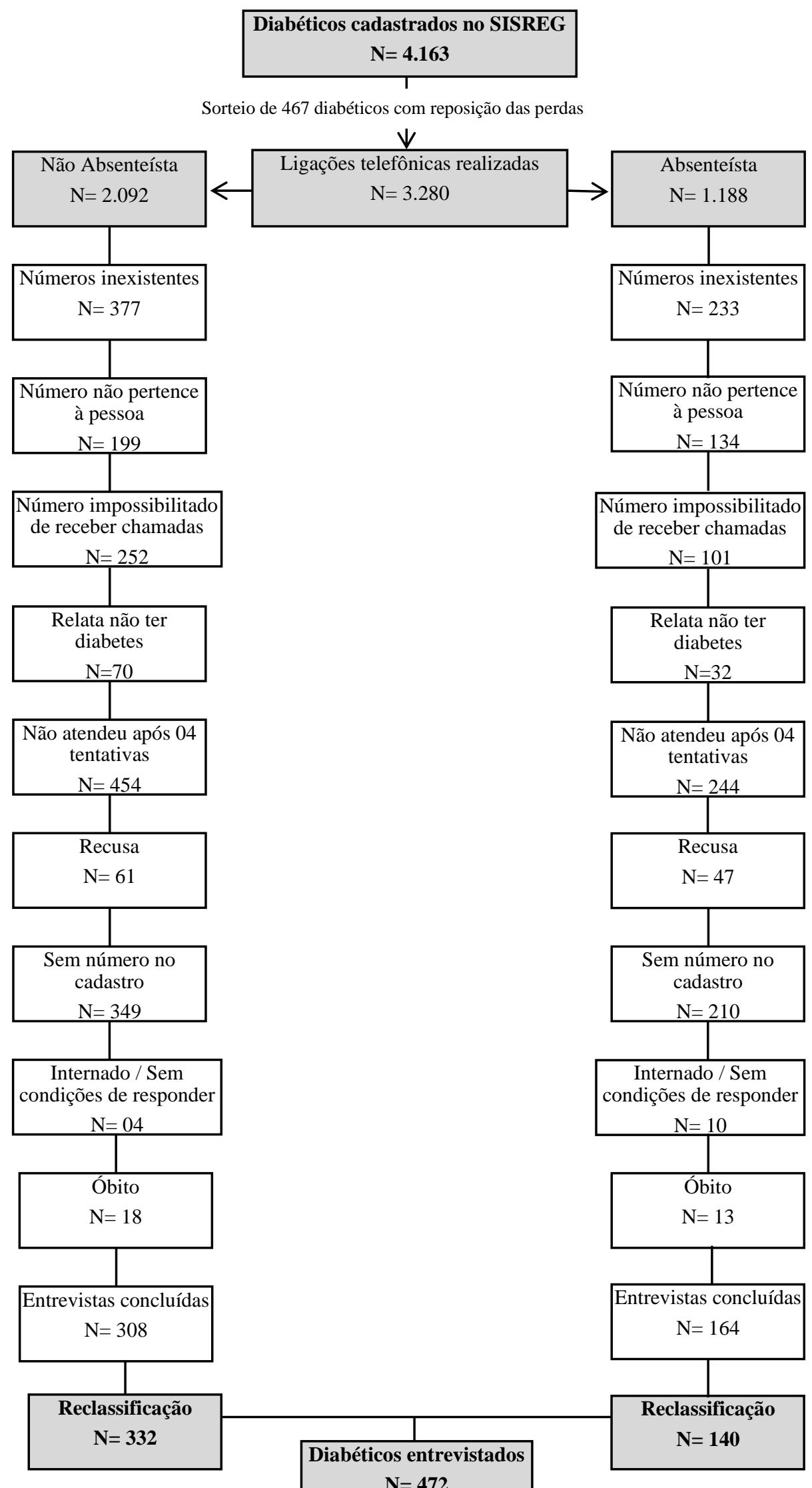


Fonte: Autores (2021).

Figura 2. Modelo Teórico hierarquizado das relações dos Determinantes Sociais do Absenteísmo dos diabéticos às consultas com endocrinologista no Sistema Único de Saúde

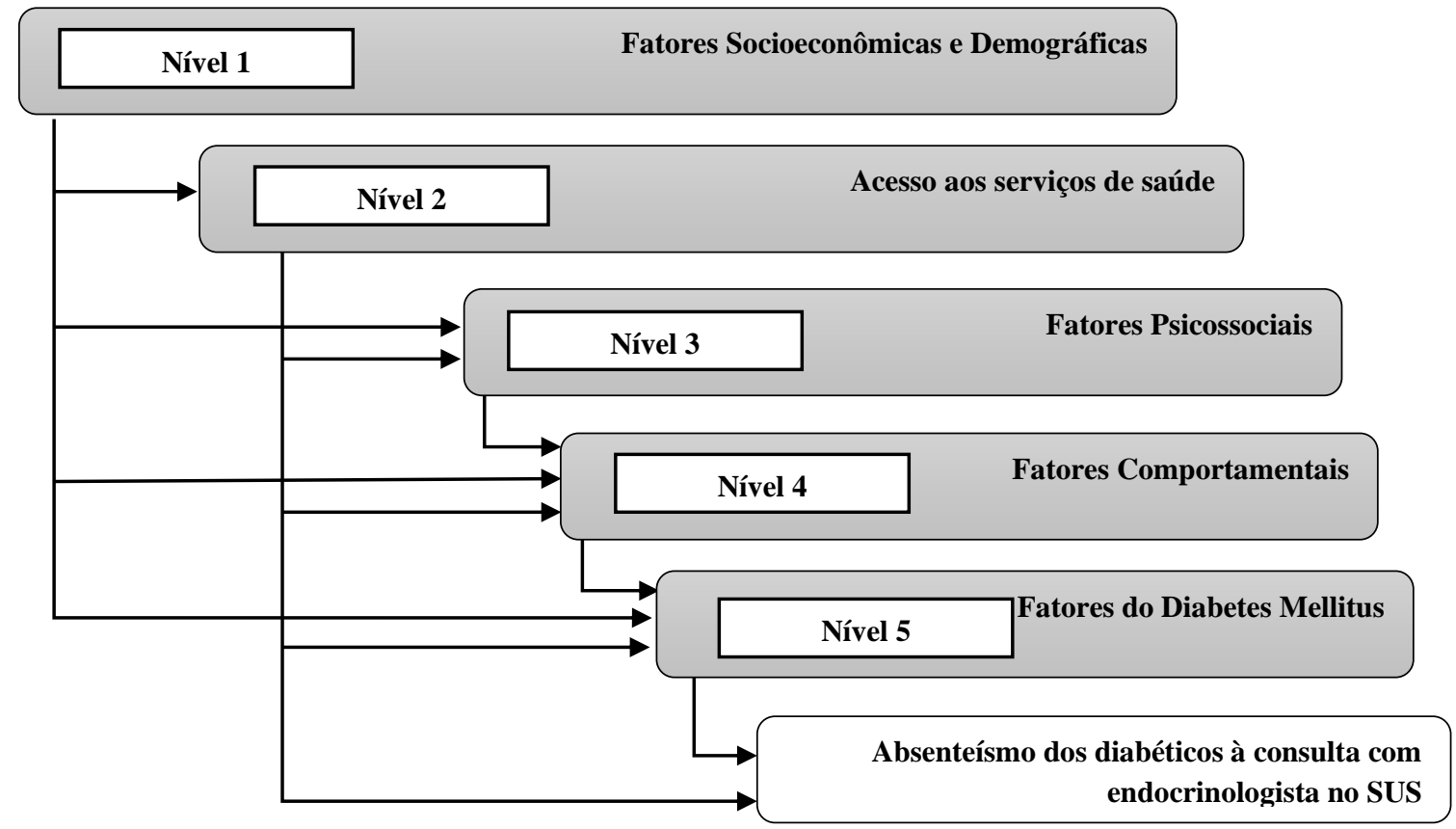

Fonte: Autores (2021).

\subsection{Análise de dados}

Para avaliar a associação entre os DSS e absenteísmo às consultas com endocrinologista foi realizada análise de regressão logística hierárquica. Foram incluídas no modelo ajustado, as variáveis que apresentaram $\mathrm{p}<0,20$ na análise bruta e mantidas no modelo final, aquelas com níveis de significância de 5\%. Ademais, foram apresentados os OR e seus respectivos intervalos de $95 \%$ de confiança e todas as variáveis foram descritas por meio das frequências absolutas e relativas.

Foram construídos cinco modelos utilizando a estratégia backward stepwise. Os OR foram estimados com ajuste estatístico para os múltiplos fatores inseridos no modelo, seguindo uma estrutura conceitual proposta por Victora, Huttly, Fuchs and Olinto (1997), em que as variáveis que integram o modelo multivariável servem de ajuste aos dados posteriormente inseridos no modelo.

Inicialmente, as variáveis do nível 1 que apresentaram $\mathrm{p}<0,20$ foram inseridas no modelo 1 e permaneceram aquelas que mantiveram $\mathrm{p}<0,005$. Em seguida, as variáveis do nível 2 foram introduzidas, simultaneamente, ao modelo 1 retendo-se no modelo 2 as variáveis que apresentaram $p<0,005$. Na sequência, incluiu-se as variáveis do nível 3 juntamente com o modelo 1 e 2 e foram mantidas as variáveis com $\mathrm{p}<0,005$ no modelo 3. Posteriormente, o mesmo procedimento foi realizado para a elaboração do modelo 4 e do modelo 5. Para todos os modelos estimou-se os OR ajustados com seus respectivos IC95\% permanecendo no modelo final as variáveis que mantiveram $\mathrm{p}<0,05$.

As variáveis do estudo foram organizadas em um banco de dados por meio do programa Epi Info 7.2.4.0 e a análise estatística foi efetuada no software Stata®, versão 14.

Este estudo foi aprovado pelo Comitê de Ética em Pesquisa com Seres Humanos do Centro de Ciências da Saúde da Universidade Federal do Espírito Santo (CEP) conforme a resolução no 466/2012, do Conselho Nacional de Ética em Pesquisa (CONEP) e Secretaria Estadual de Saúde do ES, sob o CAAE 04078918.2.0000.5060. 


\section{Resultados}

Dentre os diabéticos estudados, a maior proporção era do sexo feminino, com idade igual ou maior que 60 anos, de cor/raça branca, com menos de quatro anos de estudo, viviam com seus companheiros, residiam em área urbana, renda média familiar per capita menor que meio salário mínimo, não possuíam plano de saúde e não recebiam benefício social (bolsa família ou benefício prestação continuada) (Tabela 1). O tempo médio de espera entre a solicitação e o agendamento da consulta com o endocrinologista foi de 125,41 dias (desvio padrão: 99,58, mediana: 102, mínimo: 2, máximo: 580) (Dados não apresentados em tabela).

Na análise bruta (Tabela 2), as variáveis que em cada nível apresentaram-se associadas (p <0,20) ao absenteísmo de diabéticos às consultas com o endocrinologista foram: Nível 1 - situação conjugal, renda média domiciliar per capita e quem é o chefe da família; Nível 2 - tempo de espera em dias entre a solicitação e o agendamento da consulta com o endocrinologista e plano de saúde; Nível 3 - apoio material, apoio emocional/informação, vitimização pessoal e discriminação percebida; Nível 4 - hábito de consumir bebida alcoólica e IMC; Nível 5 - tipo de medicamento em uso e complicações do DM.

A Tabela 2 apresenta o modelo final ajustado para as variáveis dos níveis hierárquicos e permaneceram associados ao absenteísmo de diabéticos às consultas com endocrinologista os fatores socioeconômicos e demográficos, acesso aos serviços de saúde, fatores comportamentais e fatores clínicos do DM.

Verificou-se que possuir renda média domiciliar per capita maior que $\mathrm{R} \$$ 499,00 aumentou a chance de absenteísmo dos diabéticos às consultas com endocrinologista $(\mathrm{R} \$ 499,00$ a R \$ 998,00 - OR=1,668, IC95\%: 1,009-2,756; > R\$ 998,00 OR=1,702, IC95\%: 1,02-2,84), assim como, consumir bebida alcoólica (OR=1,821, IC95\%:1,031-3,239) e ser obeso (OR=1,773, IC95\%: 1,134-2,773). Observou-se que a cada um dia a menos de espera pela consulta, há uma redução de $0,03 \%$ na probabilidade de absenteísmo do diabético (OR=0,997, IC95\%:0,995-0,999). Ainda, fazer uso concomitante de hipoglicemiante oral e insulina (OR=2,2, IC95\%:1,09-4,44) e ter complicações do DM (OR=2,03, IC95\%:1,297-3,179) elevou em mais de duas vezes a chance de os diabéticos faltarem à consulta com o endocrinologista no SUS. 
Tabela 1. Distribuição, Odds ratios (OR) brutos e os respectivos intervalos de 95\% (IC95\%) entre os fatores socioeconômicos e demográficos, acesso aos serviços de saúde, fatores psicossociais, fatores comportamentais, fatores do diabetes mellitus e absenteísmo de diabéticos às consultas com endocrinologista. Brasil, Espírito Santo, 2018.

\begin{tabular}{|c|c|c|c|c|c|c|c|c|c|c|}
\hline \multirow{2}{*}{ Socioeconômico e Demográfic } & & \multicolumn{6}{|c|}{ Absenteísmo } & \multicolumn{3}{|c|}{ Análise Bruta } \\
\hline & & $\mathbf{n}$ & $\%$ & Sim & $\%$ & Não & $\%$ & OR & IC $95 \%$ & p valor \\
\hline \multirow{2}{*}{ Sexo } & Feminino & 336 & 71,19 & 105 & 31,25 & 231 & 68,75 & 1 & & \\
\hline & Masculino & 136 & 28,81 & 35 & 25,74 & 101 & 74,26 & 1,311 & $0,837-2,053$ & 0,236 \\
\hline \multirow{2}{*}{ Faixa etária } & $\geq 60$ anos & 242 & 51,27 & 75 & 30,99 & 167 & 69,01 & 1 & & \\
\hline & $20-59$ & 230 & 48,73 & 65 & 28,26 & 165 & 71,74 & 1,14 & $0,767-1,693$ & 0,516 \\
\hline \multirow{2}{*}{ Raça/Cor } & Branca & 159 & 33,69 & 44 & 27,67 & 115 & 72,33 & 1 & & \\
\hline & Preta / Parda & 313 & 66,31 & 96 & 30,67 & 217 & 69,33 & 0,864 & $0,566-1,319$ & 0,501 \\
\hline \multirow{4}{*}{ Escolaridade } & $\geq 12$ anos & 28 & 5,93 & 10 & 35,71 & 18 & 64,29 & 1 & & \\
\hline & 9 - 11 anos & 116 & 24,58 & 36 & 31,03 & 80 & 68,97 & 1,234 & $0,518-2,938$ & 0,634 \\
\hline & $5-8$ anos & 123 & 26,06 & 33 & 26,83 & 90 & 73,17 & 1,515 & $0,634-3,616$ & 0,349 \\
\hline & $\leq 4$ anos & 205 & 43,43 & 61 & 29,76 & 144 & 70,24 & 1,311 & $0,572-3,004$ & 0,521 \\
\hline \multirow{2}{*}{ Situação conjugal } & Com companheiro & 315 & 66,74 & 86 & 27,3 & 229 & 72,7 & 1 & & \\
\hline & Sem companheiro & 157 & 33,26 & 54 & 34,39 & 103 & 65,61 & 0,716 & $0,474-3,411$ & 0,113 \\
\hline \multirow{2}{*}{ Zona de moradia } & Urbana & 403 & 85,38 & 120 & 29,78 & 283 & 70,22 & 1 & & \\
\hline & Rural & 69 & 14,62 & 20 & 28,99 & 49 & 71,01 & 1,038 & $0,592-2,919$ & 0,894 \\
\hline \multirow{3}{*}{$\begin{array}{l}\text { Renda média domiciliar per } \\
\text { capita }\end{array}$} & $\leq \mathrm{R} \$ 499,00$ & 169 & 35,81 & 62 & 36,69 & 107 & 63,31 & 1 & & \\
\hline & $R \$ 499,00$ - R\$998,00 & 159 & 33,69 & 40 & 25,16 & 119 & 74,84 & 1,723 & $1,071-2,773$ & 0,025 \\
\hline & $>\mathrm{R} \$ \mathbf{9 9 8 , 0 0}$ & 144 & 30,51 & 38 & 26,39 & 106 & 73,61 & 1,616 & $0,995-2,625$ & 0,052 \\
\hline \multirow{3}{*}{ Chefe da família } & Próprio (a) & 239 & 50,64 & 78 & 32,64 & 161 & 67,36 & 1 & & \\
\hline & Cônjuge & 181 & 38,35 & 46 & 25,41 & 135 & 74,59 & 1,421 & $0,924-2,186$ & 0,109 \\
\hline & Outro & 52 & 11,02 & 16 & 30,77 & 36 & 69,23 & 1,09 & $0,570-2,083$ & 0,794 \\
\hline \multirow{4}{*}{$\begin{array}{l}\text { Escolaridade do Chefe da } \\
\text { família }\end{array}$} & $\geq 12$ anos & 29 & 6,14 & 9 & 31,03 & 20 & 68,97 & 1 & & \\
\hline & $9<12$ & 97 & 20,55 & 37 & 38,14 & 60 & 61,86 & 0,729 & $0,300-1,771$ & 0,486 \\
\hline & $5<9$ anos & 230 & 48,73 & 64 & 27,83 & 166 & 72,17 & 1,167 & $0,504-2,697$ & 0,718 \\
\hline & $\leq 4$ anos & 116 & 24,58 & 30 & 25,86 & 86 & 74,14 & 1,29 & $0,529-3,140$ & 0,575 \\
\hline \multirow{2}{*}{ Benefício Social } & Sim & 65 & 13,77 & 23 & 35,38 & 42 & 64,62 & 1 & & \\
\hline & Não & 407 & 86,23 & 117 & 28,75 & 290 & 271,25 & 1,357 & $0,781-2,357$ & 0,278 \\
\hline \multirow{2}{*}{ Água tratada } & Sim & 443 & 93,86 & 134 & 30,25 & 309 & 69,75 & 1 & & \\
\hline & Não & 29 & 6,14 & 6 & 20,69 & 23 & 79,31 & 1,662 & $0,661-4,175$ & 0,279 \\
\hline \multirow{2}{*}{ Esgoto ligado à rede } & Sim & 372 & 78,81 & 108 & 29,03 & 264 & 70,97 & 1 & & \\
\hline & Não & 100 & 21,19 & 32 & 32,00 & 68 & 68 & 0,869 & $0,540-1,399$ & 0,564 \\
\hline \multirow{2}{*}{$\begin{array}{l}\text { Coleta de lixo pelo serviço } \\
\text { público }\end{array}$} & Sim & 454 & 96,19 & 135 & 29,74 & 319 & 70,26 & 1 & & \\
\hline & Não & 18 & 3,81 & 5 & 27,78 & 13 & 72,22 & 1,1 & $0,384-3,146$ & 0,858 \\
\hline \multicolumn{11}{|l|}{ Acesso aos serviços de saúde } \\
\hline \multirow{2}{*}{ Plano de saúde } & Sim & 117 & 24,79 & 28 & 23,93 & 89 & 76,07 & 1 & & \\
\hline & Não & 355 & 75,21 & 112 & 31,55 & 243 & 68,45 & 0,682 & $0,422-1,103$ & 0,119 \\
\hline
\end{tabular}


Research, Society and Development, v. 10, n. 13, e108101320880, 2021

(CC BY 4.0) | ISSN 2525-3409 | DOI: http://dx.doi.org/10.33448/rsd-v10i13.20880

\begin{tabular}{|c|c|c|c|c|c|c|c|c|c|c|}
\hline Tempo de espera em dias & & & & & & & & 0,999 & 0,995-0,999 & 0,006 \\
\hline Psicossociais & & & & & & & & & & \\
\hline & Alto & 399 & 84,53 & 113 & 28,32 & 286 & 71,68 & 1 & & \\
\hline Apoio material & Moderado & 59 & 12,5 & 23 & 38,98 & 36 & 61,02 & 0,618 & $0,350-1,089$ & 0,096 \\
\hline & Baixo & 14 & 2,97 & 4 & 28,57 & 10 & 71,43 & 0,987 & $0,303-3,213$ & 0,984 \\
\hline Apoio emocional/ & Alto & 356 & 75,42 & 103 & 28,93 & 253 & 71,07 & 1 & & \\
\hline informação & Moderado & 98 & 20,76 & 28 & 28,57 & 70 & 71,43 & 1,017 & $0,620-1,668$ & 0,944 \\
\hline & Baixo & 18 & 3,81 & 9 & 50 & 9 & 50 & 0,407 & $0,157-1,054$ & 0,064 \\
\hline Coec̃̃o social & Alto & 17 & 3,6 & 6 & 35,29 & 11 & 64,71 & 1 & & \\
\hline Coesao social & Moderado & 297 & 62,92 & 85 & 28,62 & 212 & 71,38 & 1,36 & $0,487-3,795$ & 0,557 \\
\hline & Baixo & 158 & 33,47 & 49 & 31,01 & 109 & 68,99 & 1,213 & $0,424-3,468$ & 0,718 \\
\hline Sorunomer $>2 \mathrm{C}$ & Alto & 13 & 2,75 & 4 & 30,77 & 9 & 69,23 & 1 & & \\
\hline Segurança & Moderado & 314 & 66,53 & 97 & 30,89 & 217 & 69,11 & 0,994 & $0,298-3,307$ & 0,993 \\
\hline & Baixo & 145 & 30,72 & 39 & 26,9 & 106 & 73,1 & 1,207 & $0,351-4,417$ & 0,764 \\
\hline Vitimização pessoal & Não & 401 & 84,96 & 113 & 28,18 & 288 & 71,82 & 1 & & \\
\hline & Sim & 71 & 15,04 & 27 & 38,03 & 44 & 61,97 & 0,639 & $0,377-3,168$ & 0,096 \\
\hline Discriminação percebida & Não & 430 & 91,1 & 121 & 28,14 & 309 & 71,86 & 1 & & \\
\hline & Sim & 42 & 8,9 & 19 & 45,24 & 23 & 54,76 & 0,474 & $0,249-3,151$ & $\mathbf{0 , 0 2 3}$ \\
\hline Oulidade da caúde nercohida & Boa / Muito boa & 89 & 18,86 & 28 & 31,46 & 61 & 68,54 & 1 & & \\
\hline Qualidade da saude percebida & Regular & 213 & 45,13 & 63 & 29,58 & 150 & 70,42 & 1,092 & $0,639-1,866$ & 0,745 \\
\hline & Ruim / Muito ruim & 170 & 36,02 & 49 & 28,82 & 121 & 71,18 & 1,133 & $0,649-1,978$ & 0,659 \\
\hline Comportamentais & & & & & & & & & & \\
\hline & Nunca fumou & 254 & 53,81 & 75 & 29,53 & 179 & 70,47 & 1 & & \\
\hline Habito de fumar & Já fumou no passado, mas,parou & 189 & 40,04 & 55 & 29,1 & 134 & 70,9 & 1,02 & $0,674-1,543$ & 0,922 \\
\hline & Fuma atualmente & 29 & 6,14 & 10 & 34,48 & 19 & 65,52 & 0,796 & $0,353-1,792$ & 0,582 \\
\hline Hábito de consumir bebida & Nunca consumiu & 89 & 18,86 & 31 & 34,83 & 58 & 65,17 & 1 & & \\
\hline alcoólica & Já consumiu bebida, mas, parou & 172 & 36,44 & 54 & 31,4 & 118 & 68,6 & 1,167 & $0,679-2,008$ & 0,575 \\
\hline & Consome atualmente & 211 & 44,7 & 55 & 26,07 & 156 & 73,93 & 1,515 & $0,889-2,584$ & 0,126 \\
\hline Índice de massa corporal & $<30 \mathrm{Kg} / \mathrm{m}^{2}$ & 302 & 63,98 & 99 & 32,78 & 203 & 67,22 & 1 & & \\
\hline & $\geq 30 \mathrm{Kg} / \mathrm{m}^{2}$ & 170 & 36,02 & 41 & 24,12 & 129 & 75,88 & 1,153 & $1,002-2,348$ & 0,049 \\
\hline Do Diabetes Mellitus & & & & & & & & & & \\
\hline Tempo de DM & $<10$ anos & 172 & 36,44 & 46 & 26,74 & 126 & 73,26 & 1 & & \\
\hline & $\geq 10$ anos & 300 & 63,56 & 94 & 31,33 & 206 & 68,97 & 0,8 & $0,527-1,213$ & 0,294 \\
\hline Tino de medicamento em uso & Hipoglicemiante & 234 & 49,58 & 74 & 31,62 & 160 & 68,38 & 1 & & \\
\hline Tipo de medicamento em uso & Insulina & 164 & 34,75 & 54 & 32,93 & 110 & 67,07 & 0,942 & $0,614-1,443$ & 0,784 \\
\hline & Ambos & 74 & 15,68 & 12 & 16,22 & 62 & 83,78 & 2,389 & $1,214-4,701$ & 0,012 \\
\hline Complicações & Não & 155 & 32,84 & 59 & 38,06 & 96 & 61,94 & 1 & & \\
\hline & Sim & 317 & 67,16 & 81 & 25,55 & 236 & 74,45 & 1,79 & $1,187-2,700$ & 0,005 \\
\hline Comorbidades & Não & 178 & 37,71 & 53 & 29,78 & 125 & 70,22 & 1 & & \\
\hline & Sim & 294 & 62,29 & 87 & 29,59 & 207 & 70,41 & 1,008 & $0,671-1,156$ & 0,966 \\
\hline Limitação ou deficiência & Não & 361 & 76,48 & 108 & 29,92 & 253 & 70,08 & 1 & & \\
\hline
\end{tabular}


Research, Society and Development, v. 10, n. 13, e108101320880, 2021

(CC BY 4.0) | ISSN 2525-3409 | DOI: http://dx.doi.org/10.33448/rsd-v10i13.20880

Sim

$111 \quad 23,52$

32

28,83

79

71,17

$1,053 \quad 0,659-1,683$

0,826

Tabela 2 - Modelo hierárquico para os determinantes sociais de saúde associados ao absenteísmo de diabético às consultas com endocrinologista. Brasil, Espírito Santo, 2018

\begin{tabular}{|c|c|c|c|c|c|c|c|c|c|c|c|}
\hline & & \multicolumn{2}{|c|}{ Modelo 1} & \multicolumn{2}{|c|}{ Modelo 2} & \multicolumn{2}{|c|}{ Modelo 3} & \multicolumn{2}{|c|}{ Modelo 4} & \multicolumn{2}{|c|}{ Modelo final } \\
\hline \multicolumn{2}{|l|}{ Socioeconômico e Demográfico } & OR & IC $95 \%$ & OR & IC $95 \%$ & OR & IC $95 \%$ & OR & IC $95 \%$ & OR & $\begin{array}{c}\text { IC } \\
95 \%\end{array}$ \\
\hline \multirow{2}{*}{ Situação conjugal } & Com companheiro & 1 & & & & & & & & & \\
\hline & Sem companheiro & 0,801 & $0,482-1,329$ & & & & & & & & \\
\hline \multirow{3}{*}{$\begin{array}{l}\text { Renda média domiciliar per } \\
\text { capita }\end{array}$} & $\leq \mathrm{R} \$ 499,00$ & 1 & & 1 & & 1 & & 1 & & 1 & \\
\hline & $R \$ 499,00-R \$ 998,00$ & 1,701 & $1,055-2,744$ & 1,776 & $1,091-2,891$ & 1,804 & $1,105-2,947$ & 1,839 & $1,125-3,004$ & 1,668 & $1,009-2,756$ \\
\hline & $>\mathbf{R} \$ 998,00$ & 1,684 & $1,030-2,752$ & 1,525 & $0,929-2,505$ & 1,617 & $0,986-2,652$ & 1,659 & $1,006-2,736$ & 1,702 & $\mathbf{1 , 0 2 0 - 2 , 8 4 0}$ \\
\hline \multirow{3}{*}{ Chefe da família } & Próprio (a) & 1 & & & & & & & & & \\
\hline & Cônjuge & 1,331 & $0,812-2,183$ & & & & & & & & \\
\hline & Outro & 1,209 & $0,610-2,397$ & & & & & & & & \\
\hline \multicolumn{12}{|l|}{ Acesso aos serviços de saúde } \\
\hline \multirow{2}{*}{ Plano de saúde } & Sim & & & 1 & & & & & & & \\
\hline & Não & & & 0,719 & $0,439-1,178$ & & & & & & \\
\hline Tempo de espera em dias & & & & 0,997 & $0,995-0,999$ & 0,997 & 0,995-0,999 & 0,996 & 0,994-0,998 & 0,997 & 0,995-0,999 \\
\hline \multicolumn{12}{|l|}{ Fatores Psicossociais } \\
\hline \multirow{3}{*}{ Apoio material } & Alto & & & & & 1 & & & & & \\
\hline & Moderado & & & & & 0,636 & $0,338-1,198$ & & & & \\
\hline & Baixo & & & & & 1,433 & $0,400-5,136$ & & & & \\
\hline \multirow{3}{*}{$\begin{array}{l}\text { Apoio emocional/ } \\
\text { informação }\end{array}$} & Alto & & & & & 1 & & & & & \\
\hline & Moderado & & & & & 1,132 & $0,660-1,940$ & & & & \\
\hline & Baixo & & & & & 0,708 & $0,229-2,187$ & & & & \\
\hline \multirow{2}{*}{ Vitimização pessoal } & Não & & & & & 1 & & & & & \\
\hline & Sim & & & & & 0,643 & $0,373-1,106$ & & & & \\
\hline \multirow[t]{2}{*}{ Discriminação percebida } & Não & & & & & 1 & & & & & \\
\hline & Sim & & & & & 0,597 & $0,285-1,251$ & & & & \\
\hline \multicolumn{12}{|l|}{ Fatores Comportamentais } \\
\hline \multirow{3}{*}{$\begin{array}{l}\text { Hábito de consumir bebida } \\
\text { alcoólica }\end{array}$} & Nunca consumiu & & & & & & & 1 & & 1 & \\
\hline & $\begin{array}{l}\text { Já consumiu no passado, } \\
\text { mas, parou }\end{array}$ & & & & & & & 1,375 & $0,784-2,410$ & 1,274 & $0,714-2,274$ \\
\hline & Consome atualmente & & & & & & & 1,753 & $1,007-3,053$ & 1,828 & $1,031-3,239$ \\
\hline Índice de Massa Corporal & $<30 \mathrm{Kg} / \mathrm{m}^{2}$ & & & & & & & 1 & & 1 & \\
\hline
\end{tabular}


Research, Society and Development, v. 10, n. 13, e108101320880, 2021

(CC BY 4.0) | ISSN 2525-3409 | DOI: http://dx.doi.org/10.33448/rsd-v10i13.20880

\begin{tabular}{|c|c|c|c|c|c|}
\hline & $\geq 30 \mathrm{Kg} / \mathrm{m}^{2}$ & 1,601 & $1,035-2,475$ & $\mathbf{1 , 7 7 3}$ & $1,134-2,773$ \\
\hline \multicolumn{6}{|c|}{ Fatores do Diabetes Mellitus } \\
\hline \multirow{3}{*}{ Tipo de medicamento em uso } & Hipoglicemiante & & & 1 & \\
\hline & Insulina & & & $\mathbf{0 , 8 2}$ & $0,517-1,301$ \\
\hline & Ambos & & & 2,2 & $1,090-4,440$ \\
\hline \multirow{2}{*}{ Complicações } & Não & & & 1 & \\
\hline & Sim & & & 2,03 & $1,297-3,179$ \\
\hline
\end{tabular}

Fonte: Autores. 


\section{Discussão}

Os principais resultados evidenciam que renda média domiciliar per capita maior que $\mathrm{R} \$ 499,00$, maior tempo de espera pela consulta, consumo de bebida alcoólica, obesidade, uso concomitante de hipoglicemiante oral e insulina e ter alguma complicação do DM estão associados ao absenteísmo de diabéticos às consultas com endocrinologista no SUS.

Em relação aos fatores socioeconômicos e demográficos verificou-se que somente a renda média domiciliar per capita apresentou associação com o absenteísmo. Sendo a chance de o diabético faltar à consulta com o endocrinologista quase duas vezes maior à medida que a renda aumenta. Cabe ressaltar que a renda é uma importante medida das desigualdades em saúde na qual a posição socioeconômica dos indivíduos está relacionada às oportunidades de saúde e a possibilidade de opção entre diferentes alternativas (Barata, 2009; Solar \& Irwin, 2010).

É plausível postular que diabéticos com melhor condição socioeconômica podem realizar as consultas com o endocrinologista por outras vias que não o SUS, o que confere maior oportunidade de acessar os serviços de saúde. Haja vista que, no sistema de saúde brasileiro, coexistem a prestação de serviço exclusivamente SUS (serviços financiados e providos pelo Estado, aqui incluída a interconexão com o subsetor privado e de saúde suplementar) e a privada na qual os indivíduos utilizam o serviço via pagamento direto, copagamento ou planos de saúde (Paim, Travassos, Almeida, Bahia \& Macinko, 2011).

A PNS (2019) revelou que o principal local de atendimento para assistência médica dos diabéticos foi a unidade básica de saúde (49,6\%) seguido de consultórios particulares ou clínicas privadas (30\%) (PNS, 2020b). Dessa forma, pode-se inferir que diabéticos com baixa renda faltam menos às consultas com o endocrinologista por ser o SUS a sua única oportunidade de acessar a consulta especializada (Tesser \& Neto, 2017). Acrescenta-se, a isto, que preferencialmente a procura por serviços de saúde em indivíduos com menor renda é motivada pela doença em detrimento da prevenção (Neri \& Soares, 2002; Viacava, Oliveira, Carvalho, Laguardia \& Bellido, 2018).

Este resultado se opõe aos achados de Brewster, Bartholomew, Holt and Prince (2020) cuja revisão sistemática apontou que o baixo status socioeconômico dos diabéticos está correlacionado ao aumento do absenteísmo. Sendo o sistema de copagamento de consultas e serviços de saúde, dificuldade ou falta de transporte para o deslocamento, viver em áreas de pobreza, complicações e problemas psicossociais, alguns dentre os diversos fatores implicados nessa relação. Entretanto, devese considerar que essas pesquisas foram realizadas com sistemas de saúde distintos de este estudo, o que inviabilizou comparações.

O acesso aos serviços de saúde no Brasil é mais influenciado pela renda do que por outras variáveis que refletem a condição socioeconômica (Travassos, Oliveira \& Viacava, 2006). Há uma relação direta entre renda domiciliar per capita e acesso às consultas médicas, assim, à medida que os rendimentos se tornam mais elevados, aumenta a frequência de consultas médicas (PNS 2020b). Porém, no Brasil não foram encontrados estudos que analisassem a relação da renda com o absenteísmo na atenção ambulatorial especializada (AAE), tampouco com os diabéticos.

Outro ponto relevante é que o tratamento do DM gera um alto custo financeiro e social ao diabético e a sua família decorrente dos gastos com a monitorização da glicemia (glicosímetro e fitas), medicamentos, insulina regular e/ou especiais, seringas, dieta específica, dentre outros (Seuring, Archangelidi \& Shurcke, 2015). A vista disso, é possível inferir que quanto menor a renda, maior é a dependência do SUS, ainda, quanto maior os custos com o DM maior a necessidade de acesso ao SUS. Isto se deve às políticas de enfrentamento às doenças crônicas no país como os programas de assistência farmacêutica, apoio diagnóstico e promoção da saúde, por exemplo, apesar do desafio à sustentabilidade das ações frente ao ineficiente financiamento da saúde (Malta, et al., 2017)

No Brasil, o tempo de espera, como determinante intermediário, está atrelado à oferta e demanda de serviços e é considerado uma das principais barreiras ao acesso a cuidados de saúde, principalmente, na AAE (Bender, Molina \& Mello, 
2010). Tempo de espera consiste uma barreira organizacional relacionada ao serviço de saúde, dificulta a conduta e o manejo do diabético na APS, fragmenta a assistência e o cuidado, além de contribuir para o agravamento e as complicações do DM, o que pode ecoar em todos os níveis de atenção (Salci, Meirelles \& Silva, 2017, Low, Khoo, Tavintharan, Lim \& Sum, 2016).

O longo tempo de espera por uma consulta com o especialista, neste estudo o endocrinologista, não é um problema exclusivo do sistema de saúde brasileiro e afeta diversos países. Diante disso, depreende-se que a longa espera pode desencadear fatores como o esquecimento, a desistência, a busca por outros meios de acesso e perda da necessidade (Bender, Molina \& Melo, 2010; Mesa, Asencio \& Ruiz, 2015). Ainda, piora no curso da doença, busca por serviços de urgência e emergência (Cavalcanti, Cavalcanti, Serrano, Santana, 2013; Bittar, Magalhães, Martines, Felizola \& Falcão, 2016) e recrudescimento das internações hospitalares (Low, Khoo, Tavintharan, Lim \& Sum, 2016; Karter, et al., 2004), dentre outros motivos que se inter-relacionam e contribuem para altas taxas de absenteísmo.

Destaca-se que devido à diversidade das metodologias empregadas nos estudos que avaliaram o absenteísmo, não foi possível identificar um padrão para o intervalo de tempo de espera pela consulta especializada. Dessa forma, qualquer comparação dos resultados dever ser realizada com cautela (Conill, Giovanella \& Almeida, 2011; Dantas, Fleck, Oliveira, Hamacher, 2018; Rodrigues, et al., 2019; Farias, Giovanella, Oliveira, Santos Neto, 2019; Mesa, Asencio \& Ruiz, 2015; Vieira, Lima \& Gazzinelli, 2015; Pinto, Soranz, Scardua \& Silva, 2017; Baduy, Feuerwerker, Zucoli \& Borian, 2011; Low, Khoo, Tavintharan, Lim \& Sum, 2016).

Fatores comportamentais como os hábitos alimentares, prática de atividade física, tabagismo e consumo de álcool são importantes determinantes de saúde e, quando desigualmente distribuídos entre os diferentes níveis socioeconômicos, podem contribuir como potenciais determinantes das desigualdades em saúde (Comissão Nacional dos Determinantes Sociais Da Saúde, 2008).

No que se refere ao DM, já está bem documentado que o consumo de álcool prejudica o controle da doença de forma que interfere na ação da insulina, aumenta o risco de hipoglicemia e promove o ganho de peso. Da mesma maneira, a obesidade tem sido associada a pior perfil lipídico, a elevados níveis pressóricos e à presença de síndrome metabólica (IDF, 2019; Malta, et al., 2017; Pietraszek, Gregersen \& Hermansen, 2010; Vasquez, et al., 2007). Diante disso, os resultados de este estudo mostraram que o consumo de bebida alcoólica e a obesidade no diabético aumentaram a probabilidade de absenteísmo à consulta com o endocrinologista. O que sugere um padrão de comportamento não saudável possivelmente influenciado pela estrutura socioeconômica, cultura e as relações de pertencimento aos grupos sociais nos quais operam os determinantes estruturais das iniquidades em saúde (Ferreira, Bispo, Casotti \& Braga, 2013).

No Brasil, pesquisa referiu que o consumo de bebida alcoólica é maior entre indivíduos com renda e nível de escolaridade elevados (PNS, 2020a). Todavia, os estudos que analisaram essa relação apresentaram discordâncias, o que sugere heterogeneidade de definições, instrumentos e pontos de corte, fato que dificulta realizar inferências (Moura \& Malta, 2011; Ferreira, Bispo, Casotti \& Braga, 2013).

Nesse sentido, é importante considerar que para a maioria dos diabéticos a necessidade de mudanças para um estilo de vida saudável envolvem processos que podem causar depressão e ansiedade (Broadbent, Donkin \& Stroh, 2011; Teixeira, et al., 2014). Assim como o consumo excessivo de álcool e a obesidade podem afetar a autoestima, a autoimagem, assim como, gerar transtornos de interação social e incapacidade funcional (Sociedade Brasileira de Diabetes, 2019).

Por outro lado, é possível especular que as intervenções necessárias à prevenção e promoção da saúde, tanto na APS quanto na AAE, sejam insipientes e fragmentadas. Pois, o Ministério da Saúde preconiza que as linhas de cuidado à pessoa com DM organizem o itinerário do indivíduo em suas necessidades pela rede de serviços e qualifiquem as portas de entrada do sistema, de forma que os profissionais de saúde possam acolher, compreender, corresponsabilizar, intervir com vínculo, produzir autonomia e um atendimento adequado e oportuno (Marinho, et al., 2011). A falta dificulta a longitudinalidade e 
integralidade do cuidado, bem como, prejudica a intervenção adequada, oportuna e as ações preventivas (Low, Karter, et al., 2004; Khoo, Tavintharan, Lim \& Sum, 2016).

No Brasil tem sido observado grandes avanços relacionados as ações, programas de prevenção e promoção a saúde aos portadores de doenças crônicas não transmissíveis, mas, ainda persiste o desafio de romper com o modelo biomédico e articular ações intersetoriais de forma humanizada e equitativas (Malta, et al., 2014).

Com relação aos fatores clínicos do DM, fazer uso de insulina associada a hipoglicemiante oral e ter alguma complicação da doença aumenta em mais de duas vezes a chance de absenteísmo. Assim como, estudo realizado por Karter, et al. (2004) comparando diabéticos que realizaram dieta com os que utilizaram tratamento medicamentoso verificaram que a chance de absenteísmo aumentou em 1,4 para aqueles em uso de hipoglicemiantes orais e 1,7 para aqueles em uso de insulina. Além disso, foi 74\% maior entre os diabéticos que obtiveram alto índice de comorbidade (Karter, et al., 2004). Da mesma forma, Zailinawati, Ng \& Nik-Sherina (2015) verificaram que pacientes com mais de quatro doenças crônicas tiveram 54\% maior probabilidade de faltar as consultas.

Sabe-se que para o controle do DM é imprescindível alcançar e manter os níveis glicêmicos tão próximos da normalidade quanto viável, o que pode ser obtido por meio da adequada adesão ao tratamento e mudanças no estilo de vida. Entretanto, neste estudo, considerando que quase a metade dos diabéticos tem idade entre 20 e 59 anos, pressupõe-se que, devido as suas ocupações laborais, disponham de pouco tempo para se dedicarem às ações relacionadas à prevenção das complicações e promoção à saúde, como consultas periódicas e a prática de atividade física.

Sob esse aspecto, outra hipótese explicativa é que o absenteísmo pode ocorrer como consequência da percepção que os diabéticos tem a respeito da sua saúde, seja pela desinformação ou incompreensão da doença, seja pela resignação advinda do maior tempo de convivência com a doença. Situações que podem impactar negativamente o autocuidado e adesão ao tratamento, bem como o acompanhamento regular nos serviços de saúde (Broadbent, Donkin \& Stroh, 2011; Borges, Gorayeb $\&$ Foss-Freitas, 2013).

O DM é uma condição crônica de curso longo e permanente que exige respostas e ações contínuas, proativas e integradas do sistema de atenção. Diante de tal afirmação, era esperado que o diabético obeso, em uso de hipoglicemiante oral acrescido da insulina e com complicações tivesse maior utilização dos serviços de saúde, principalmente na AAE, haja vista, que esses fatores já demonstram uma evolução ruim da doença. Em contraponto, o absenteísmo também pode contribuir para o agravamento das condições de saúde do diabético, uma vez que não correspondem adequadamente ao plano terapêutico. Estas situações podem acarretar graves repercussões sociais como maior frequência de internações hospitalares, licenças, limitações físicas, aposentadoria precoce e mortalidade (Leijten, et al., 2014).

Neste estudo, os fatores psicossociais não permaneceram no modelo final, apesar das variáveis apoio material, apoio emocional/informação, vitimização pessoal e discriminação percebidas apresentarem significância na análise bruta.

Este estudo apresenta algumas limitações como a possibilidade de viés de informação decorrente da diferença temporal entre a coleta dos dados e o desfecho e das informações autorreferidas - renda per capita domiciliar e IMC. Entretanto, como uma medida de controle de qualidade os entrevistadores foram treinados e avaliados periodicamente. Outra limitação é referente ao banco de dados do SISREG no que diz respeito a impossibilidade de contactar os indivíduos elegíveis para o estudo pela desatualização ou inexistência dos dados telefônicos.

Por fim, entende-se que absenteísmo às consultas com o endocrinologista no SUS está relacionado, principalmente, à condição socioeconômica do diabético como determinante estrutural das desigualdades em saúde, pois, pode configurar padrões diferenciados de vulnerabilidade e exposição aos determinantes intermediários - diferenças na oferta e organização dos serviços de saúde como barreira de acesso à AAE, maior gravidade da condição de saúde e piores hábitos de vida. O que 
gera um círculo vicioso entre o absenteísmo e os seus determinantes, promovendo a mobilidade social (deslocamento dos indivíduos por meio de um sistema de estratificação social) e o incremento das desigualdades sociais em saúde.

\section{Conclusão}

Nos diabéticos, os determinantes sociais de saúde, maior renda média domiciliar, mais tempo de espera pela consulta, atual consumo de bebida alcoólica, obesidade, uso conjunto de hipoglicemiante oral e insulina e complicações do DM estão associados ao absenteísmo às consultas com o endocrinologista no SUS.

O modelo apresenta evidências para avaliação de prioridades e implementação da rede de atenção às pessoas com DM, assim como subsídios para a reformulação das linhas de cuidado para essa população no ES de forma a minimizar as altas taxas de absenteísmo na AAE.

O conhecimento dos DSS relacionados ao absenteísmo de diabéticos no SUS permitiram compreender os processos que produzem e medeiam as desigualdades em saúde, o que é imprescindível para que seja possível buscar formas de enfrentamento, sejam no campo das políticas públicas, sejam no campo das vivências.

Dada a natureza e complexidade do comportamento absenteísta, bem como dos determinantes sociais de saúde, são necessários mais estudos que analisem a singularidade das necessidades dos indivíduos em cada especialidade e a integralidade e longitudinalidade das redes de atenção à saúde regionalizadas e hierarquizadas.

\section{Referências}

Baduy, R. S., Feuerwerker, L. C. M., Zucoli, M. \& Borian, J. T. (2011). Healthcare regulation and healthcare management as tools to assure comprehensiveness and equity in health. Cadernos Saúde Pública, 27(2), 295-304. https://doi.org/10.1590/S0102-311X2011000200011

Barata, R. B. O que queremos dizer com desigualdades sociais em saúde? (2009) In: Como e por que as desigualdades sociais fazem mal à saúde (pp. 11-21). FIOCRUZ.

Beltrame, S. M. Oliveira, A. E., Santos, M. A. B. Dos \& Santos Neto, E. T. (2019). Absenteísmo de usuários como fator de desperdício: Desafio para sustentabilidade em sistema universal de saúde. Saúde Debate, 43(123), 1015-1030. https://doi.org/10.1590/0103-1104201912303

Bender, A. S., Molina, L. R. \& Mello, A. L. S. F. (2010) Absenteísmo na Atenção Secundária e suas implicações na Atenção Básica. Revista Espaço para a Saúde, 11(2), 56-65. https://doi.org/10.22421/15177130-2010v11n2p56

Bittar, O. J. N. V., Magalhães, A., Martines, C. M., Felizola, N. B. G. \& Falcão, L. H. B. (2016). Absenteísmo em atendimento ambulatorial de especialidades no estado de São Paulo. Boletim Epidemiológico Paulista, 13(152), 19-32.

Borges, A. M., Gorayeb, R. \& Foss-Freitas, M. C. (2013) Caracterização psicossocial de pacientes diabéticos de um hospital público universitário. Arquivos brasileiro de psicologia, 65(2), 214-229.

Brasil. (2013). Diretrizes para o cuidado das pessoas com doenças crônicas nas redes de atenção à saúde e nas linhas de cuidado prioritárias. Ministério da Saúde, Secretaria de Atenção à Saúde, Departamento de Atenção Básica. https://bvsms.saude.gov.br/bvs/publicacoes/diretrizes\%20_ cuidado_pessoas\%20_doencas_cronicas.pdf

Brewster, S., Bartholomew, J., Holt, R. I. G. \& Price, H. (2020). Non-attendance at diabetes outpatient appointments: a systematic review. Diabetic Medicine, 37, 1427-1442. https://doi.org/10.1111/dme.14241

Broadbent, E., Donkin, L. \& Stroh, J. C. (2011). Illness and treatment perceptions are associated with adherence to medications, diet, and exercise in diabetic patients. Diabetes Care, 34(2), 338-340 https://doi.org/10.2337/dc10-1779

Cavalcanti, R. P., Cavalcanti, J. C. M., Serrano, R. M. S. M. \& Santana, P. R. (2013). Absenteísmo de consultas especializadas nos sistema de saúde público: relação entre causas e o processo de trabalho de equipes de saúde da família, João Pessoa - PB, Brasil. Revista Tempus Actas de Saúde Coletiva, 7(2).

Comissão Nacional Sobre Determinantes Sociais de Saúde (2008). As causas das iniquidades em saúde no Brasil. https://bvsms.saude.gov.br/bvs/publicacoes/causas_sociais_iniquidades.pdf

Conill, E. M., Giovanella, L. \& Almeida, P. F. de (2011). Waiting lists in public systems: From expanding supply to timely access? Revista Ciência \& Saúde Coletiva, 16(6), 2783-2794. https://doi.org/10.1590/S1413-81232011000600017

Costa, A. F., Flor, L. S., Campos, M. R., Oliveira, A. F. de, Costa, M. F. S., Silva, R. S. da, Lobato, L. C. P. \& Schramm, J. M. A. (2017). Carga do diabetes mellitus tipo 2 no Brasil. Cadernos de Saúde Pública. 33(2), e00197915. https://doi.org/10.1590/0102-311X00197915 
Danaei, G., Lu, Y., Singh, G. M., Carnahan, E., Stevens, G. A., Cowan, M. J., Farzadfar, F., Lin, J. K., Finucane, M. M., Rao, M., Khang, Y-H., Riley, L. M., Mozaffarian, D., Lim, S. S., \& Ezzati, M. (2014). Cardiovascular disease, chronic kidney disease, and diabetes mortality burden of cardiometabolic risk factors from 1980 to 2010: A comparative risk assessment. Lancet Diabetes Endocrinology, 2, 634-647. http://dx.doi.org/10.1016/S2213-8587(14)70102-0

Dantas, L. F., Fleck, J. L., Oliveira, F. L. C. \& Hamacher, S. (2018). No-shows in appointment scheduling - a systematic literature review. Health Policy, 122(4), 412-421. https://doi.org/10.1016/j.healthpol.2018.02.002

Farias, C. M. L., Giovanella, L., Oliveira, A. E. \& Santos Neto, E. T. et al. (2019). Tempo de espera e absenteísmo na atenção especializada: um desafio para os sistemas universais de saúde. Saúde Debate, 43(5), 190-204.

Farias, C. M. L., Santos Neto, E. T., Esposti, C. D. D. (2020). Absenteísmo de usuários: barreiras e determinantes no acesso aos serviços de saúde. Revista Brasileira de Medicina de Família e Comunidade, 15(42), 2239. https://doi.org/10.1590/0103-11042019S516

Ferreira, L. N., Bispo, J. P. Jr. (2013). Prevalência e fatores associados ao consumo abusivo e à dependência de álcool. Revista Ciência \& Saúde Coletiva, 18(11) 3409-3418. https://doi.org/10.1590/S1413-81232013001100030

Griep, R. H., Chor, D., Faerstein, e., Werneck, G. L. \& Lopes, C. S. (2005). Validade de constructo de escala de apoio social do Medical Outcomes Study adaptada para o português no Estudo Pró-Saúde. Cadernos de Saúde Pública, 21(3), 703-714. https://doi.org/10.1590/S0102-311X2005000300004

Griep, R. H., Chor, D., Faerstein, E. \& Lopes, C. (2003). Apoio social: confiabilidade teste-reteste de escala no Estudo Pró-Saúde. Cadernos de Saúde Pública, 19, 625-34. https://doi.org/10.1590/S0102-311X2003000200029

International Diabetes Federation. IDF Diabetes Atlas Ninth edition 2019. International Diabetes Federation. 2019.

Karter, A. J., Parker, M. M., Moffet, H. H., Ahmed, A. T. Ferrara, A., Liu, J. Y. \& Selby, J. V. (2004). Missed appointments and poor glycemic control: an opportunity to identify high-risk diabetic patients. Medical Care, 42(2), 110-5. https://doi.org/10.1097/01.mlr.0000109023.64650.73

Leijten, F. R., van den Heuvel, S. G., Ybema, J. F., van der Beek, A. J., Robroek, S. J., \& Burdorf, A. (2014). The influence of chronic health problems on work ability and productivity at work: a longitudinal study among older employees. Scandinavian journal of work, environment \& health, 40(5), 473-482. https://doi.org/10.5271/sjweh.3444

Leopardi, M. T., Beck, C. L. C., Nietsche, E. A. \& Gonzales, R. M. B. Metodologia da pesquisa na saúde. Santa Maria: Pallotti, 2001.

Low, S. K., Khoo, J. K., Tavintharan, S., Lim, S. C., \& Sum, C. F. (2016). Missed Appointments at a Diabetes Centre: Not a Small Problem. Annals of the Academy of Medicine, Singapore, 45(1), 1-5.

Malta, D. C., Silva, M. M. A. da, Moura, L. de \& Morais Neto, O. L. de (2017). A implantação do Sistema de Vigilância de Doenças Crônicas Não Transmissíveis no Brasil, 2003 a 2015: alcances e desafios. Revista Brasileira de Epidemiologia, 20(4), 661-675. https://doi.org/10.1590/19805497201700040009

Malta, D. C., Bernal, R. T. I., Iser, B. P. M., Szwarcwald, C. L., Duncan, B. B. \& Schmidt, M. I. (2017). Fatores associados ao diabetes autorreferido segundo a Pesquisa Nacional de Saúde, 2013. Revista de Saúde Pública, 51(Suppl. 1), 12s. https://doi.org/10.1590/S1518-8787.2017051000011

Malta, D., M., Moura, L. de, Prado, R. R. do, Escalante, J. C., Schimidt, M. I. \& Duncan, B. B. (2014). Mortalidade por doenças crônicas não transmissíveis no Brasil e suas regiões, 2000 a 2011. Epidemiologia e Serviços de Saúde, 23(4), 599-608. https://doi.org/10.5123/S1679-49742014000400002

Marinho, C. C. C., Conceição, C. S., Santos, M. L. M. F. dos, Carvalho, S. S. M. de, Menezes, T. M.O. \& Guimarães, E. P (2011). O olhar de uma equipe multiprofissional sobre as linhas de cuidado: (Vi)vendo o tecer dos fios. Revista baiana de saúde pública, 35(3), 619-633. https://doi.org/10.22278/23182660.2011.v35.n3.a282

Mesa, M. L. J., Asencio, J. M. M. \& Ruiz, F. R. (2015). Factores determinantes y coste económico del absentismo de pacientes en consultas externas de la Agencia Sanitaria Costa del Sol. Anales del sistema sanitario de Navarra, 38(2). https://dx.doi.org/10.4321/S1137-66272015000200007

Moura, E. C., Malta, D.C. Consumo de bebidas alcoólicas na população adulta brasileira: características sociodemográficas e tendência. Revista Brasileira de Epidemiologia, 14(Suppl. 1), 61-70. https://doi.org/10.1590/S1415-790X2011000500007

Mujahid, M. S., Diez Roux, A. V., Morenoff, J. D., \& Raghunathan, T. (2007). Assessing the measurement properties of neighborhood scales: from psychometrics to ecometrics. American journal of epidemiology, 165(8), 858-867. https://doi.org/10.1093/aje/kwm040

Neri, M.; Soares, W. (2002). Desigualdades social e saúde no Brasil. Cadernos de Saúde Pública, 18(Suppl.), 62-70. https://doi.org/10.1590/S0102$311 \mathrm{X} 2002000700009$

Organização Mundial de Saúde. (2011). Diminuindo as diferenças: A prática das políticas sobre determinantes sociais da saúde: Documento de Discussão. Conferência Mundial sobre Determinantes Sociais da Saúde. https://dssbr.ensp.fiocruz.br/wp-content/uploads/2020/10/Documento-Tecnico-da-Conferenciavers\%C3\%A3o-final.pdf

Paim, J., Travassos, C., Almeida, C., Bahia, L. \& Macinko, J. (2011). O sistema de saúde brasileiro: história, avanços e desafios. Saúde no Brasil. Lancet, 1, 11-31. https://doi.org/10.1016/S0140-6736(11)60054-8

Pesquisa Nacional de Saúde. (2020b). 2019: Informações sobre domicílios, acesso e utilização dos serviços de saúde. Brasil e Grandes Regiões/IBGE, Coordenação de Trabalho e Rendimento. Instituto Brasileiro de Geografia e Estatística. https://biblioteca.ibge.gov.br/visualizacao/livros/liv101748.pdf

Pesquisa Nacional de Saúde. (2020a). 2019: percepção do estado de saúde, estilos de vida, doenças crônicas e saúde bucal. Brasil e grandes regiões/IBGE, Coordenação de Trabalho e Rendimento. https://biblioteca.ibge.gov.br/visualizacao/livros/liv101764.pdf

Pietraszek, A., Gregersen, S., \& Hermansen, K. (2010). Alcohol and type 2 diabetes. A review. Nutrition, metabolism, and cardiovascular diseases: NMCD, 20(5), 366-375. https://doi.org/10.1016/j.numecd.2010.05.001 
Pinto, L. F. Soranz, D., Scardua, M. T. \& Silva, I. M. (2017). Ambulatory municipal regulation of the unified health system services in Rio de Janeiro: Advances, limitations and challenges. Ciência \& Saúde Coletiva, 22(4),1257-1267. https://doi.org/10.1590/1413-81232017224.26422016

Rodrigues, J. G., Stein, J. O. S., Nunes, A. T., Vasconcelos, K. A. Zndonade, E. \& Anhoque. (2019). Perfil de absenteísmo às consultas eletivas de subespecialidades pediátricas de um hospital universitário. Revista brasileira de pesquisa em saúde, 21(3), 113-121.

Salci, M. A., Meirelles, B. H. S., Silva, D. M. V. G. (2017). Prevenção das complicações crônicas do diabetes mellitus à luz da complexidade. Revista Brasileira de Enfermagem, 70(5), 996-1003. https://doi.org/10.1590/0034-7167-2016-0080

Seuring, T., Archangelidi, O., \& Suhrcke, M. (2015). The Economic Costs of Type 2 Diabetes: A Global Systematic Review. PharmacoEconomics, 33(8), 811-831. https://doi.org/10.1007/s40273-015-0268-9

Sherbourne, C. D., \& Stewart, A. L. (1991). The MOS social support survey. Social science \& medicine (1982), 32(6), 705-714. https://doi.org/10.1016/02779536(91)90150-b

Sociedade Brasileira de Diabetes. (2020). Diretrizes Sociedade Brasileira de Diabetes 2019-2020. http://www.saude.ba.gov.br/wpcontent/uploads/2020/02/Diretrizes-Sociedade-Brasileira-de-Diabetes-2019-2020.pdf

Solar, O. \& Irwin, A. (2010). A conceptual framework for action on the social determinants of health. Social Determinants of Health. Discussion Paper 2 (Policy and Practice). Organização Mundial de Saúde. https://www.who.int/sdhconference/resources/ConceptualframeworkforactiononSDH_eng.pdf

Teixeira, C. R. S., Arrelias, C. C. A., Zanetti, A. C. G., Gonela, J. T., Miyar, 1. \& Franco, R. C. (2014). Consumo de álcool e problemas emocionais relacionados ao diabetes mellitus. Revista Eletrônica de Saúde Mental Álcool e Drogas, 10(1), 11-16. https://doi.org/10.11606/issn.1806-6976.v10i1p11-16

Tesser, C. D.; Neto, P. P. (2017). Atenção especializada ambulatorial no Sistema Único de Saúde: para superar um vazio. Ciência \& Saúde Coletiva, 22(3), 941-951. https://doi.org/10.1590/1413-81232017223.18842016

Travassos, C.; Oliveira, E. X. G. de; Viacava, F. (2006). Desigualdades geográficas e sociais no acesso aos serviços de saúde no Brasil: 1998 e 2003. Ciência \& Saúde Coletiva, v. 11(4), 975-986. https://doi.org/10.1590/S1413-81232006000400019

Turkcan, A., Nuti, L.A., DeLaurentis, P., Tian, Z., Daggy, J., Zhang, L., Lawley, M., \& Sands, L. (2013). No-Show Modeling for Adult Ambulatory Clinics. In: Denton, B. (eds), Healthcare Operations Management. International Series in Operations Research \& Management Science, 184, 251-288. https://doi.org/10.1007/978-1-4614-5885-2_10

Vasquez, A. C. J. Pereira, P. F., Gomide, R. M., Batista, M. C. R., Campos, M. T. F. S., Sant'Ana, L. F. R., Rosado, L. E. F. P. L. \& Priore, S. E. (2007). Influência do Excesso de Peso Corporal e da Adiposidade Central na Glicemia e no Perfil Lipídico de Pacientes Portadores de Diabetes Mellitus Tipo 2. Arquivos Brasileiros de Endocrinologia, 51(9). https://doi.org/10.1590/S0004-27302007000900015

Viacava, F., Oliveira, R. A. D. de, Carvalho, C. C., Laguardia, J. \& Bllido, J. G. (2018). SUS: oferta, acesso e utilização de serviços de saúde nos últimos 30 anos. Ciência \& Saúde Coletiva, 23(6), 1751-1762. https://doi.org/10.1590/1413-81232018236.06022018

Victora, C. G., Huttly, S. R., Fuchs, S. C., \& Olinto, M. T. (1997). The role of conceptual frameworks in epidemiological analysis: a hierarchical approach. International journal of epidemiology, 26(1), 224-227. https://doi.org/10.1093/ije/26.1.224

Vieira, E. W. R.; Lima, T. M. N.; Gazzinelli, A. (2015). Tempo de espera por consulta médica especializada em um município de pequeno porte de Minas Gerais, Brasil. Revista Mineira de Enfermagem, 19(1). 65-71. https://doi.org/10.5935/1415-2762.20150006

World Health Organization Expert Committee. (1995). Physical Status. Phisical status: The use and interpretation of anthropometry. World Health Organization technical report series, 854, 1-452.

Zailinawati, A. H., Ng, C. J., \& Nik-Sherina, H. (2006). Why do patients with chronic illnesses fail to keep their appointments? A telephone interview. AsiaPacific journal of public health, 18(1), 10-15. https://doi.org/10.1177/101 